\title{
Subcutaneous mass of the right thigh
}

\author{
Christos Voucharas, Dimitrios Papaioannidis, Konstantinos Papamichael
}

A 50-year-old woman presented with a 5-cm diameter mass of rubbery consistency, loosely adherent to the skin, not tender, not adherent to the underlying tissues, located in the medeal site of the right thigh. The patient was first aware of the mass 10 years ago, when it was $1 \mathrm{~cm}$ in diameter. She lived in a rural area and had a dog as a pet. Laboratory examinations were within normal range. The mass was surgically removed (figure 1), and then opened and turned inside out (figure 2).

\section{Arta General Hospital, 47100 Arta, Greece Department of \\ Surgery \\ C Voucharas \\ K Papamichael \\ Department of \\ Medicine \\ D Papaioannidis}

Correspondence to $\mathrm{Chr}$

Voucharas, Sourmenon 10 54636 Thessaloniki, Greece

Accepted 20 August 1997

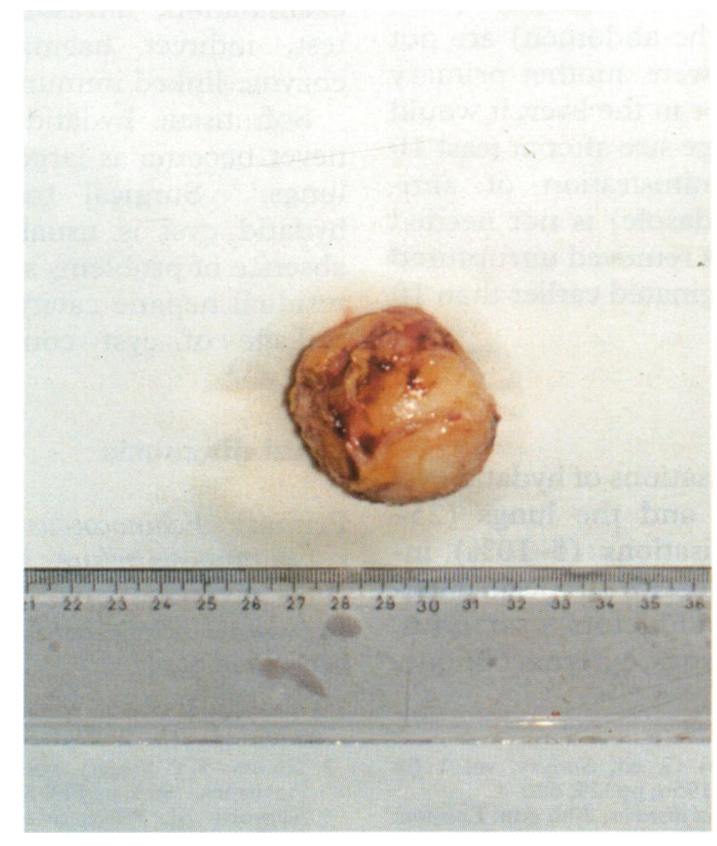

Figure 1

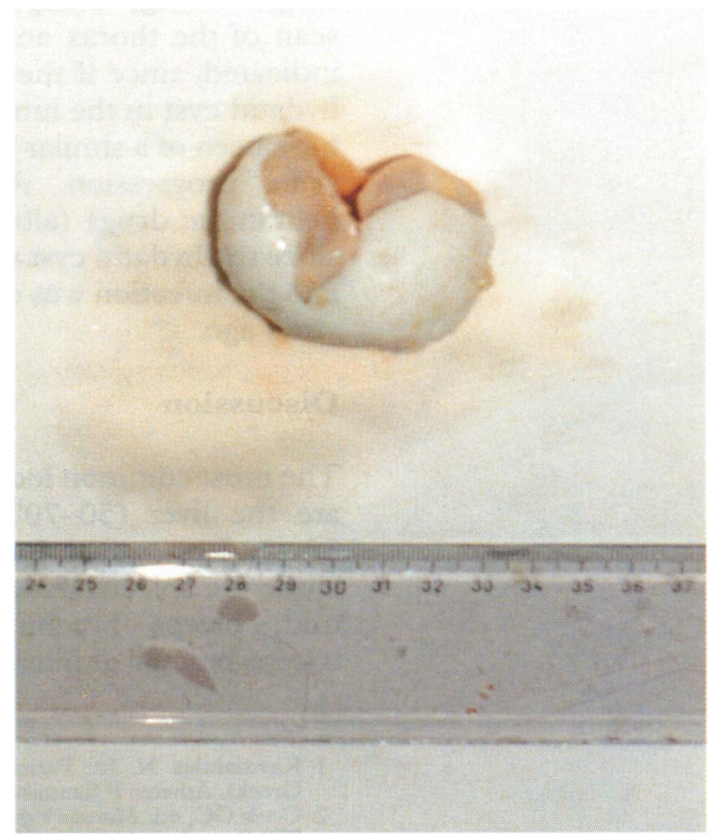

Figure 2

\section{Questions}

1 What is the diagnosis and the differential diagnosis?

2 What other investigations are indicated in this patient?

3 Is there any additional procedure required? 


\section{Answers}

QUESTION 1

The diagnosis is primary hydatid cyst in the subcutaneous tissue. When the cyst was turned inside out, the inner germinate membrane was visible (figure 2). The differential diagnosis is sebaceous cyst, lipoma, fibroma, histiocytoma, sarcoma (with or without cystic devolution), lymphoma, tuberculosis abscess, aneurysm, pelvic hernia, and hydatid cyst. ${ }^{1}$

QUESTION 2

Pathologic examination confirmed the diagnosis, which was considered at first sight after surgical excision. An X-ray of the thorax and the abdomen, and ultrasonography of the abdomen should also be performed. Specific examinations supplying more a detailed description (eg, computed tomography (CT) scan of the thorax and the abdomen) are not indicated, since if there were another primary hydatid cyst in the lung or in the liver, it would have been of a similar large size after at least 10 years progression. Administration of antihelminthic drugs (albendasole) is not needed since the hydatid cyst was removed unruptured and the infection was originated earlier than 10 years ago.

\section{Discussion}

The most common localisations of hydatid cyst are the liver (50-70\%) and the lungs (25$30 \%)$. Less usual localisations $(5-10 \%)$ include the spleen, skin, muscles (trapezius, deltoid, biceps brachii, adductors, sartorius, triceps brachii, gluteus, psoas, external oblique,

1 Kavazarakis N. In: Parisianos G, ed, Surgery, vol 1 (in Greek). Athens: P Jiannakou, 1956; pp325, 620-4.

2 Cook GC, ed. Manson's tropical diseases, 20th edn. London: WB Saunders, 1996; pp1486-94. rectus abdominis, temporalis), bones, peritoneal cavity, kidneys, brain, thyroid, heart, breasts, eye, pancreas, parotid gland, ovarium, cavernous sinus. ${ }^{1-3}$

Primary hydatid cysts in subcutaneous tissue are very rare. No report could be found in the Greek or international literature in the last 40 years. Hydatid cysts in extrahepatic locations are mainly primary (the oncosphere is transported either through the portal vein and then through the liver capillaries or directly through the lymph vessels of the gastrointestinal track to target organs), or less commonly secondary after rupture of a cyst (spontaneous or iatrogenic). ${ }^{2-4}$ Experimentally, it has been shown that echinococcus eggs can cause hydatid cysts after being inoculated in subcutaneous tissue. ${ }^{1}$

Pre-operative diagnosis relies on $\mathrm{X}$-ray examination, ultrasound, CT, Casoni's skin test, indirect haemagglutination test, and enzyme-linked immunoassay (ELISA). ${ }^{2-4}$

Soft tissue hydatid cysts grow slowly and never become as large as those in the liver or lungs. ${ }^{13}$ Surgical treatment of soft tissue hydatid cyst is usually easy because of the absence of problems such as management of a residual hepatic cavity, intrabiliary rupture or spillage of cyst content in the peritoneal cavity. ${ }^{34}$

\section{Final diagnosis}

Primary Echinococcus granulosus cyst in the subcutaneous tissue.

Keywords: echinococcosis; hydatid cyst; soft tissue benign tumours

3 Tountas KT. Surgery, vols 2 \& 3. (in Greek). Athens: G Parisianos, 1975; pp443-50, 775-82.

4 Schwartz SJ. Principles of surgery, 6th edn. New York: McGraw Hill, 1994; pp1332-3. 